\title{
Doubly Charged Ion Spectra in Mass Spectra of Hydrocarbons
}

\author{
By Fred L. Mohler, Evelyn G. Bloom, Edmund J. Wells, Jr., Jonathan H. Lengel, ${ }^{1}$ and \\ C. Edward Wise
}

\begin{abstract}
A compilation of doubly charged ions in the mass spectra of 148 hydrocarbons has been made, based on mass spectra published in the American Petroleum Institute Catalog of Mass Spectral Data. In hydrocarbons with three, four, or five carbon atoms, the most probable double ionization process involves loss of all but two or three hydrogen atoms without breaking carbon bonds. Unsaturated molecules give larger doubly charged ion peaks than saturated molecules, and ions with an even number of hydrogen atoms tend to be more abundant than those with an odd number. In saturated hydrocarbons with six or more carbon atoms, double ionization with breaking of carbon bonds is most probable, but in benzenes and highly unsaturated molecules, double ionization without loss of carbon atoms is probable. These heavier molecules show distinctive differences depending on molecular structure.

The ratio of ion current at 70 volts to that at 50 volts ionizing voltage is an indication of the appearance potential of the ion. It increases as the number of hydrogen atoms removed increases, and the results indicate that hydrogen atoms are removed in pairs to give $\mathrm{H}_{2}$.
\end{abstract}

\section{Introduction}

The mass spectra of hydrocarbons and other polyatomic molecules in general show some small peaks that arise from doubly charged ions. Ions with a double charge are characterized by an apparent mass equal to half the molecular weight of the ion. If the molecular weight is an odd number, the doubly charged ion will be distinguished by half-integer values; if it is an even number, then it will often happen that the apparent mass of the doubly charged ion is coincident with the mass peak of a singly charged ion. For this reason the data on the occurrence of doubly charged ions are necessarily incomplete.

The application of the mass spectrometer to chemical analysis has resulted in the careful measurement of mass spectra of at least 200 hydrocarbons; and these spectra afford a vast

\footnotetext{
${ }^{1}$ Now at Naval Powder Factory, Indian Head, Md.
}

amount of data on the existence of doubly charged ions, but no attempt has previously been made to collect and correlate these data. The Catalog of Mass Spectral Data of the American Petroleum Institute [1] ${ }^{2}$ has been the source of data used in this compilation, and the tables contributed by the Mass Spectrometry Section of the National Bureau of Standards have been used except in cases noted in the text. There are distinct advantages in using data of one laboratory insofar as possible, for there are minor differences between laboratories arising from instrumental differences and different methods of operation. In three cases, ethane [2], ethylene [3], and benzene [4], appearance potentials of some doubly charged ions have been published. For most molecules all that is known is the relative intensity of the doubly charged ions at one or two ionizing voltages.

2 Figures in brackets indicate the literature references at the end of this paper. 


\section{Experimental Procedure}

Mass spectra have been measured with a $180^{\circ}$ Consolidated mass spectrometer following recommended procedures [5]. The instrument is equipped with automatic electron current control, and the ionization chamber is thermostatically maintained at $245^{\circ} \mathrm{C}$. The compounds used are in most cases the pure NBS Standard Samples.

The Catalog of Mass Spectral Data [1] gives intensities of mass peaks relative to the maximum peak taken as 100 . The limit of this instrument is about 0.01 percent of the maximum deflection, and as spectra are always obtained with a pressure sufficient to give a nearly full scale deflection at the maximum, the lower limit remains about the same in all spectra. Peaks of doubly charged ions rarely exceed 1 percent of the maximum, and most of the peaks are less than 0.1 percent. The reproducibility on these small peaks is about 10 percent of the peak height, or 0.01 percent of the maximum for peaks less than 0.1 percent.

The tables in the catalog give relative intensities with ionizing voltages of both 50 and 70 volts. This paper tabulates values at 70 volts and includes some data on the ratio of ion current at 70 volts to that at 50 volts. The relative intensity at 70 volts is greater than at 50 volts and often much greater. The API Catalog gives spectra from several laboratories for many of the compounds, and in general there is good agreement on these small doubly charged ion peaks in all cases where they are reported.

\section{Results}

\section{1. $\mathrm{C}_{2}$ Hydrocarbons and Methane}

No doubly charged ions in the mass range 6 to 8 are observed in methane. Table 1 lists the doubly charged ions in $\mathrm{CH}_{3} \cdot \mathrm{CH}_{3}, \mathrm{CH}_{2}=\mathrm{CH}_{2}$ and $\mathrm{CH} \equiv$ CH. The doubly charged ions all contain two carbon atoms, and those ions of even molecular weight are coincident with the singly charged ions $\mathrm{CH}^{+}, \mathrm{CH}_{2}{ }^{+}$and $\mathrm{CH}_{3}{ }^{+}$. However, in two cases there is a basis for estimating the intensity of doubly charged ions of even molecular weight. Kusch, Hustrulid, and Tate [3] report a small peak in ethylene at mass $14 \frac{1}{2}$ from the ion $\mathrm{C}^{12} \mathrm{C}^{15} \mathrm{H}_{4}{ }^{++}$. The $\mathrm{C}_{2}^{12} \mathrm{H}_{4}{ }^{++}$peak will be about 50 times as large as the $14 \frac{1}{2}$ peak. They used a mass spectrometer of greater sensitivity than the Consolidated instrument. Similarly, in acetylene a peak has been observed at $13 \frac{1}{2}$ from the ion $\mathrm{C}^{12} \mathrm{C}^{13} \mathrm{H}_{2}^{++}$, and the $\mathrm{C}_{2}^{12} \mathrm{H}_{2}{ }^{++}$peak is computed from this. The distinctive differences between the three $\mathrm{C}_{2}$ compounds are of a type not found in the heavier hydrocarbons.

TABLE 1. Doubly charged ions of $\mathrm{C}_{2}$ hydrocarbons

Relative intensities in percentage of maximum peak in spectrurn

\begin{tabular}{|c|c|c|c|c|c|}
\hline Compound & $\mathrm{C}_{2} \mathrm{H}$ & $\mathrm{C}_{2} \mathrm{H}_{2}$ & $\mathrm{C}_{2} \mathrm{H}_{3}$ & $\mathrm{C}_{2} \mathrm{H}_{4}$ & $\mathrm{C}_{2} \mathrm{H}_{5}$ \\
\hline Ethane & . & ....... & 0.07 & & 0.70 \\
\hline Ethylene..... & ${ }^{1} 0.007$ & 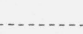 & .42 & ${ }^{1} 0.3$ & \\
\hline Acetylene.... & - & ${ }^{2} 3.9$ & & & \\
\hline
\end{tabular}

1 Kusch, Hustrulid, and Tate, Phys. Rev. 52, 843 (1937). The value for $\mathrm{C}_{2} \mathrm{H}_{4}++$ is an estimate based on a small peak at $141 / 2$ from $\mathrm{C}^{12} \mathrm{C}^{13} \mathrm{H}_{4}{ }^{++}$.

2 The value for $\mathrm{C}_{2} \mathrm{H}_{2}++$ is based on a peak at $131 / 2$ from $\mathrm{C}^{12} \mathrm{C}^{13} \mathrm{H}_{2}++$.

\section{2. $\mathrm{C}_{3}$ Hydrocarbons}

Table 2 lists doubly charged ions found in the five hydrocarbons $\mathrm{CH}_{3} \cdot \mathrm{CH}_{2} \cdot \mathrm{CH}_{3}, \mathrm{CH}_{2} \cdot \mathrm{CH}_{2} \cdot \mathrm{CH}_{2}$, $\mathrm{CH}_{2}=\mathrm{CH}-\mathrm{CH}_{3}, \quad \mathrm{CH}_{2}=\mathrm{C}=\mathrm{CH}_{2}, \quad$ and $\mathrm{CH}_{3}-$ $\mathrm{C} \equiv \mathrm{CH}$. All observed doubly charged ions contain three carbon atoms, and the apparent masses fall in a mass range 19 to 21 containing no singly charged ions. Delfosse and Bleakney [6] have previously published data on doubly charged ions in propane, propylene, and propadiene at 100 -volts ionizing voltage.

TABLE 2. Doubly charged ions of $\mathrm{C}_{3}$ hydrocarbons

Relative intensities in percentage of maximum peak in spectrum

\begin{tabular}{|c|c|c|c|c|c|}
\hline Compound & $\mathrm{C}_{3} \mathrm{H}_{2}$ & $\mathrm{C}_{3} \mathrm{H}_{3}$ & $\mathrm{C}_{3} \mathrm{H}_{4}$ & $\mathrm{C}_{3} \mathrm{H}_{5}$ & $\mathrm{C}_{3} \mathrm{H}_{6}$ \\
\hline Propane & 1. 10 & 0.61 & 1. 12 & 0.26 & 0.02 \\
\hline Cyclopropane... & 3.33 & 1.44 & 2.40 & .73 & .02 \\
\hline Propylene....... & 3. 23 & 1.96 & 2. 35 & .67 & .04 \\
\hline Propadiene $1 \ldots$ & 2. 79 & 3.20 & 3.61 & -..... & ...... \\
\hline Propyne $1 \ldots$ & 3.23 & 2.34 & 0.80 & -..... & ...... \\
\hline
\end{tabular}

1 From unpublished mass spectra at a 50-volt ionizing potential.

With one exception, the relative intensity of the ions $\mathrm{C}_{3} \mathrm{H}_{2}{ }^{++}, \mathrm{C}_{3} \mathrm{H}_{3}{ }^{++}$and $\mathrm{C}_{3} \mathrm{H}_{4}{ }^{++}$are similar in the five molecules in contrast to some distinctive differences in the spectra of the singly charged ions. Propyne differs from the others in that the $\mathrm{C}_{3} \mathrm{H}_{4}{ }^{++}$ peak is small, and this is of interest because otherwise the mass spectra of propyne and propadiene are nearly identical. 


\section{3. $\mathrm{C}_{4}$ Hydrocarbons}

Table 3 lists the doubly charged ions in ten $\mathrm{C}_{4}$ hydrocarbons. $\mathrm{C}_{2} \mathrm{H}_{n}{ }^{+}$ions of mass 25 to 29 mask doubly charged $\mathrm{C}_{4}$ ions of even molecular weight, but in 1,3-butadiene a small peak at $27 \frac{1}{2}$ from $\mathrm{C}^{13} \mathrm{C}_{3}^{12} \mathrm{H}_{6}{ }^{++}$permits a rough estimate of the intensity of the $\mathrm{C}_{4}^{12} \mathrm{H}_{6}{ }^{++}$peak.

TABLE 3. Doubly charged ions of $\mathrm{C}_{4}$ hydrocarbons

Relative intensities in percentage of maximum peak in spectrum

\begin{tabular}{|c|c|c|c|c|c|c|c|c|}
\hline Compound & $\mathrm{C}_{3} \mathrm{H}_{2}$ & $\mathrm{C}_{3} \mathrm{H}_{3}$ & $\mathrm{C}_{3} \mathrm{H}_{4}$ & $\mathrm{C}_{4} \mathrm{H}$ & $\mathrm{C}_{4} \mathrm{H}_{3}$ & $\mathrm{C}_{4} \mathrm{H}_{5}$ & $\mathrm{C}_{4} \mathrm{H}_{6}$ & $\mathrm{C}_{4} \mathrm{H}_{7}$ \\
\hline$n$-Butane $\ldots . . .$. & 0.04 & 0.01 & 0.02 & .... & 0.36 & 0.08 & .... & 0.05 \\
\hline$i$-Butane ........ & .05 & .02 & .04 & $\ldots$ & .21 & .02 & $\ldots$ & -...- \\
\hline 1-Butene....... & .06 & .03 & .02 & -... & 1.06 & .17 & $\ldots$ & .06 \\
\hline cis-2-Butene..... & .02 & .01 & .01 & .... & 1. 55 & .27 & .... & .17 \\
\hline trans-2-Butene.. & $\ldots$ & $\ldots$ & -... & $\ldots$ & 1.65 & .29 & .... & .27 \\
\hline$i$-Butene $\ldots . . .$. & .06 & .03 & .03 & -... & 0.95 & .09 & -... & $\cdots$ \\
\hline 1,2-Butadiene... & .03 & .02 & -... & $\ldots$ & 4.07 & .60 & $\ldots$ & $\ldots$ \\
\hline 1,3-Butadiene... & .09 & .02 & $\ldots$ & $\ldots$ & 3. 99 & .48 & ${ }^{1} 1.60$ & -... \\
\hline 1-Butyne........ & .06 & .05 & $\ldots$ & .03 & 3.62 & .20 & .... & .... \\
\hline 2-Butyne......... & $\ldots$ & .... & .... & .01 & 3.25 & .46 & $\ldots$ & -... \\
\hline
\end{tabular}

1 Estimated from a small peak of $\mathrm{C}^{13} \mathrm{C}_{3}{ }^{12} \mathrm{H}_{6}{ }^{++}$.

The relative intensities are roughly similar in all these spectra, and there is no evident relation between the intensity distribution in the mass spectra of doubly charged and singly charged ions. $\mathrm{C}_{4} \mathrm{H}_{3}{ }^{++}$is in every case the largest peak, and the height is least in $\mathrm{C}_{4} \mathrm{H}_{10}$ isomers, greater in the $\mathrm{C}_{4} \mathrm{H}_{8}$ isomers, and largest in $\mathrm{C}_{4} \mathrm{H}_{6}$ molecules. There is a small but real difference between the cis- and trans-2-butene (the singly charged ion spectra are very similar).

\section{4. $\mathrm{C}_{5}$ Hydrocarbons}

Table 4 lists doubly charged ions of $12 \mathrm{C}_{5}$ hydrocarbons. It includes all $\mathrm{C}_{5} \mathrm{H}_{12}$ and $\mathrm{C}_{5} \mathrm{H}_{10}$ isomers except some rare cyclics but only two of the many $\mathrm{C}_{5} \mathrm{H}_{8}$ isomers. As in the case of $\mathrm{C}_{4}$ and $\mathrm{C}_{3}$ hydrocarbons, the relative intensities in all these spectra are similar. $\mathrm{C}_{5} \mathrm{H}_{2}{ }^{++}$is the largest doubly charged peak and it is smallest in $\mathrm{C}_{5} \mathrm{H}_{12}$ isomers, larger in $\mathrm{C}_{5} \mathrm{H}_{10}$ isomers, and largest in the $\mathrm{C}_{5} \mathrm{H}_{8}$ isomers. Ions with three or four carbon atoms give small peaks often near the threshold for observation. Ions with an even number of hydrogen atoms tend to be more abundant than those with an odd number. This is probably a fairly general rule for doubly charged ions, partially concealed by the fact that there is often masking of ions with a double charge and an even number of $\mathrm{H}$ atoms.

TABLE 4. Doubly charged ions of $\mathrm{C}_{5}$ hydrocarbons

\begin{tabular}{|c|c|c|c|c|c|c|c|c|c|c|c|c|c|}
\hline Compound & $\mathrm{C}_{3} \mathrm{H}_{2}$ & $\mathrm{C}_{3} \mathrm{H}_{3}$ & $\mathrm{C}_{3} \mathrm{H}_{4}$ & $\mathrm{C}_{4} \mathrm{H}_{3}$ & $\mathrm{C}_{5} \mathrm{H}$ & $\mathrm{C}_{5} \mathrm{H}_{2}$ & $\mathrm{C}_{5} \mathrm{H}_{3}$ & $\mathrm{C}_{5} \mathrm{H}_{4}$ & $\mathrm{C}_{5} \mathrm{H}_{5}$ & $\mathrm{C}_{5} \mathrm{H}_{6}$ & $\mathrm{C}_{5} \mathrm{H}_{7}$ & $\mathrm{C}_{5} \mathrm{H}_{8}$ & $\mathrm{C}_{5} \mathrm{H}_{9}$ \\
\hline$n$-Pentane & -... & -...- & .... & 0.04 & -...- & 0.33 & 0.08 & 0.13 & 0.05 & 0.07 & 0.02 & ...- & -..- \\
\hline$i$-Pentane....... & -... & -... & -... & .06 & -... & .27 & .05 & .09 & .02 & .03 & -... & -... & -... \\
\hline Neopentane..... & -... & -..- & --.- & .04 & $-\cdots$ & .09 & .02 & .04 & .01 & -.-- & -..- & -..- & $\cdots-$ \\
\hline Cyclopentane..... & $-\cdots$ & -.-- & -..- & ---- & 0.01 & .84 & .19 & .44 & .16 & .24 & .05 & 0.01 & -...- \\
\hline 1-Pentene....... & 0.02 & 0.01 & 0.01 & .03 & .01 & 1.03 & .24 & .34 & .21 & .22 & .10 & .04 & 0.02 \\
\hline cis-2-Pentene_....... &...- & -... & -... & .06 & .03 & 1.23 & .37 & .53 & .25 & .22 & .09 & .08 & .02 \\
\hline trans-2-Pentene ${ }^{1} \ldots$ & .01 & .... & .01 & .06 & .01 & 1.52 & .37 & .53 & .26 & .24 & .10 & .09 & .03 \\
\hline 2-me-1-Butene & 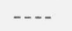 &.--- & $-\cdots$ & .06 & .01 & .90 & .21 & .29 & .15 & .13 & .06 & .04 & .01 \\
\hline 3-me-1-Butene 1 ............. & -... & -...- & -..- & .10 & .01 & .55 & .14 & .15 & .08 & .06 & .04 & -... & -... \\
\hline 2-me-2-Butene-_............ & .01 & $-\cdots$ & .01 & .04 & .02 & 1.15 & .25 & .39 & .17 & .29 & .07 & .12 & .02 \\
\hline Cyclopentene $\ldots . . . . . .$. & .03 & .02 & .01 & .02 & .05 & 2.43 & .64 & 1.00 & .70 & .74 & .35 & .13 & -... \\
\hline 2-me-1,3-Butadiene ${ }^{2}-\ldots$ & .03 & .01 & .01 & .15 & .10 & 4.31 & 1.07 & 1.07 & .80 & .85 & .30 & .41 & -..- \\
\hline
\end{tabular}

1 These also have a small peak at $\mathrm{C}_{4} \mathrm{H}_{5}++$.

2 Also has a peak $\mathrm{C}_{5}{ }^{++0.08}$.

\section{5. $\mathrm{C}_{6}$ Hydrocarbons}

Table 5 lists $23 \mathrm{C}_{6}$ hydrocarbons including all $\mathrm{C}_{6} \mathrm{H}_{14}$ isomers and most of the common $\mathrm{C}_{6} \mathrm{H}_{12}$ isomers but only two $\mathrm{C}_{6} \mathrm{H}_{10}$ molecules. Doubly charged $\mathrm{C}_{6}$ ions with an even number of hydrogen atoms are masked by singly charged ions. These spectra in contrast to $\mathrm{C}_{5}, \mathrm{C}_{4}$, and $\mathrm{C}_{3}$ molecules show considerable variations and do not follow the rule that double ionization without loss of a carbon atom is most probable. $\mathrm{C}_{6}$ doubly charged ions are missing in $\mathrm{C}_{6} \mathrm{H}_{14}$ isomers and are relatively weak in the olefins. The intensity distribution 
TABLE 5. Doubly charged ions of $\mathrm{C}_{6}$ hydrocarbons

Relative intensities in percentage of maximum peak in spectrum

\begin{tabular}{|c|c|c|c|c|c|c|c|c|c|c|c|c|c|}
\hline Compound & $\mathrm{C}_{4} \mathrm{H}_{3}$ & $\mathrm{C}_{5} \mathrm{H}_{2}$ & $\mathrm{C}_{5} \mathrm{H}_{3}$ & $\mathrm{C}_{3} \mathrm{H}_{4}$ & $\mathrm{C}_{5} \mathrm{H}_{5}$ & $\mathrm{C}_{5} \mathrm{H}_{6}$ & $\mathrm{C}_{5} \mathrm{H}_{7}$ & $\mathrm{C}_{5} \mathrm{H}_{8}$ & $\mathrm{C}_{5} \mathrm{H}_{8}$ & $\mathrm{C}_{6} \mathrm{H}_{3}$ & $\mathrm{C}_{6} \mathrm{H}_{5}$ & $\mathrm{C}_{6} \mathrm{H}_{7}$ & $\mathrm{C}_{6} \mathrm{H}_{0}$ \\
\hline n-Hexane............. & 0.02 & 0.07 & 0.02 & 0.06 & 0.03 & 0.05 & 0.02 & 0.01 &.-- & -..- & $-\ldots$ & $-\ldots$ & $-\ldots$ \\
\hline 2-me-Pentane & .02 & .08 & .02 & .05 & .03 & .06 & .03 & .02 & 0.02 & -...- & -... & -... & -...- \\
\hline 3-me-Pentane & .02 & .08 & .03 & .06 & .03 & .03 & $-\ldots$ & --- & $-\cdots$ & $-\cdots$ & -... & $-\ldots$ &.-- \\
\hline 2,2-me - -Butane & .02 & .03 & .03 & .07 & .02 & .03 & .03 & .03 & .01 & $-\ldots$ & $-\cdots$ & -..- &.-- \\
\hline 2,3-me - Butane & .01 & .06 & .01 & .04 & .02 & .02 & .02 & .02 &..- &..- & $\ldots$ &..-- & $-\ldots$ \\
\hline Cyclohexane........ & .03 & .06 & .... & .06 & .02 & .04 & -..- &.-- & -..- & $\ldots$ & 0.01 & 0.07 & 0.03 \\
\hline me-Cyclopentane & $\ldots$ & .12 & .02 & .08 & .06 & .07 & .03 & $-\cdots$ & $-\cdots$ & $-\ldots$ & $\ldots$ & .02 & $\ldots$ \\
\hline 1-Hexene............ & .01 & .15 & .04 & .09 & .05 & .03 & .02 & -... & -... & .06 & -... & - - - & ...- \\
\hline cis-2-Hexene-_. & .02 & .20 & .04 & .12 & .02 & .02 & .01 & -..- & $-\ldots$ & .05 & -..- & .02 & $-\ldots$ \\
\hline trans-2-Hexene & .02 & .14 & .04 & .10 & .02 & .02 & .01 & -...- & $\ldots$ & .05 & -... & .01 & .... \\
\hline trans-3-Hexene & .02 & .28 & .09 & .14 & .11 & .24 & .15 & .02 & .42 & .09 & .01 & .04 & .03 \\
\hline 3-me-1-Pentene & .02 & .18 & .05 & .10 & .07 & .04 & .02 &.-- &.-- & --- &.-- & $-\ldots$ & $-\ldots$ \\
\hline 4-me-1-Pentene & -... & .17 & .05 & .18 & .13 & .17 & .13 & .03 & .07 & .01 & -... & ...- & -... \\
\hline 2-me-2-Pentene & .02 & .19 & .05 & .09 & .05 & .03 & .02 & $-\ldots$ & $\ldots$ & .03 & -..- & .05 & .05 \\
\hline 3-me-cis-2-Pentene & .01 & .14 & .04 & .12 & .04 & .03 & .01 & $-\ldots$ & $-\ldots$ & .02 & $-\ldots$ &.$\theta 4$ & .02 \\
\hline 3-me-trans-2-Pentene & .01 & .19 & .04 & .10 & .04 & .03 & .02 & $-\ldots$ & -..- & .03 & $-\ldots$ & .05 & .03 \\
\hline 4-me-cis-2-Pentene. & $-\ldots$ & .32 & .09 & .16 & .11 & .09 & .07 & .02 & .02 & .01 & $-\ldots$ & -..- & . \\
\hline 4-me-trans-2-Pentene & -... & .29 & .09 & .17 & .12 & .09 & .07 & .02 & .02 & .01 & -... & $\ldots$ & -... \\
\hline 2-eth-1-Butene & .04 & .23 & .05 & .10 & .04 & .04 & .03 & $-\ldots$ & .02 & .03 & -..- & .03 & $\ldots$ \\
\hline 3,3-mer-1-Butene & .02 & .18 & .06 & .07 & .06 & .02 & .04 & $-\ldots$ & $\ldots$ & $-\ldots$ & $-\ldots$ & $-\ldots$ & $-\ldots$ \\
\hline 2,3-me 2 -2-Butene & $-\cdots$ & .17 & .05 & .07 & .02 & .02 & $-\cdots$ & $-\ldots$ & $-\cdots$ & .01 & $-\cdots$ & .01 & .08 \\
\hline Cyclohexene...... & .03 & .11 & .02 & .05 & .02 & -..- & $-\ldots$ & $-\ldots$ & --- & .25 & .11 & .53 & .09 \\
\hline 1,5-Hexadiene ${ }^{1}$ & .03 & .14 & .04 & .05 & .03 & -..- & --- & $-\ldots$ & --- & .07 & .02 & .05 & $-\cdots$ \\
\hline
\end{tabular}

1 This also has peaks $\mathrm{C}_{3} \mathrm{H}_{2}++0.03, \mathrm{C}_{3} \mathrm{H}_{3}{ }^{++} 0.02, \mathrm{C}_{3} \mathrm{H}_{4}{ }^{++} 0.02, \mathrm{C}_{3} \mathrm{H}_{5}{ }^{++} 0.01$.

TABLE 6. Doubly charged ions of $\mathrm{C}_{7}$ hydrocarbons

Relative intensities in percentage of maximum peak in spectrum

\begin{tabular}{|c|c|c|c|c|c|c|c|c|c|c|c|c|c|c|c|c|}
\hline Compound & $\mathrm{C}_{5} \mathrm{H}_{2}$ & $\mathrm{C}_{5} \mathrm{H}_{3}$ & $\mathrm{C}_{5} \mathrm{H}_{4}$ & $\mathrm{C}_{5} \mathrm{H}_{5}$ & $\mathrm{C}_{5} \mathrm{H}_{6}$ & $\mathrm{C}_{5} \mathrm{H}_{7}$ & $\mathrm{C}_{5} \mathrm{H}_{8}$ & $\mathrm{C}_{5} \mathrm{H}_{9}$ & $\mathrm{C}_{3} \mathrm{H}_{10}$ & $\mathrm{C}_{6} \mathrm{H}_{7}$ & $\mathrm{C}_{6} \mathrm{H}_{9}$ & $\mathrm{C}_{7} \mathrm{H}_{6}$ & $\mathrm{C}_{7} \mathrm{H}_{7}$ & $\mathrm{C}_{7} \mathrm{H}_{8}$ & $\mathrm{C}_{7} \mathrm{H}_{9}$ & $\mathrm{C}_{7} \mathrm{H}_{10}$ \\
\hline$n$-Heptane & 0.03 & -.... & 0.02 & -..... & 0.02 & ...... & ..... & ..... & ..... & -.... & ..... & ..... & -.... & 0.02 & -.... & $\ldots$ \\
\hline 2-me-Hexane & .02 & -...- & .02 & -...- & .02 & ..... & -.... & -.... & $\ldots$ & ..... & 0.02 & ..... & -.... & .01 & $\ldots$ & $\ldots$ \\
\hline 3-me-Hexane .............. & .03 & $\ldots$ & .03 & $-\ldots$ & .02 & $\ldots$ & $-\ldots$ & $-\ldots$ & $\ldots$ & $\ldots$ & $\ldots$ & -...- & -...- & .01 & $\ldots$ & $-\cdots$ \\
\hline 3-eth-Pentane & .02 & ..... & ..... & -...- & ..... & $\ldots$ & ..... & -.... & $\ldots$ & -... & $\ldots$ & ..... & -.... & .01 & ..... & -... \\
\hline 2,2-mer-Pentane & .02 & 0.01 & .03 & 0.01 & .02 & $\ldots-$ & -...- & -...- & $-\ldots$ & $\ldots$ & .07 & $-\cdots$ & $-\ldots$ & -...- & $\ldots$ & $\cdots$ \\
\hline 2,3-mes-Pentane & .02 & $\ldots$ & .04 & $\ldots$ & .01 & $-\ldots$ & -...- & $-\ldots$ & -...- & $\ldots$ & $-\cdots$ & -... & -...- & -...- & -.... & 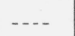 \\
\hline 2,4-mes-Pentane & .03 & $\ldots$ & .02 & .01 & .09 & 0.05 & 0.05 & 0.09 & 0.06 & $-\ldots$ & .02 & -.... & -...- & .01 & $-\ldots$ & $-\cdots$ \\
\hline $3,3-\mathrm{me}_{2}$-Pentane & .03 & .01 & .02 & $-\ldots$ & -...- & $-\ldots$ & $-\ldots$ & $-\ldots$ & $\ldots$ & -...- & $-\cdots$ & $\cdots$ & -...- & $-\ldots$ & -.... & -... \\
\hline 2,2,3-mes-Butane & .02 & -...- & $: 03$ & 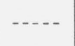 & .02 & -...- & n... & $-\ldots-$ & $-\ldots$ & $-\ldots$ & .03 & $\cdots$ & $-\ldots$ & $\cdots$ & -...- & $-\cdots$ \\
\hline me-Cyclohexane ${ }^{1} \ldots$ & .03 & ..... & .03 & ..... & .04 & $-\ldots$ & .04 & $\ldots$. & .03 & 0.05 & $\ldots-$ & 0.20 & 0.06 & .26 & 0.02 & 0.10 \\
\hline eth-Cyclopentane & .08 & .01 & .02 & -.... & .02 & , n... & .01 & -.... & -.... & .01 & .02 & .16 & .05 & .19 & .02 & .02 \\
\hline 1,1-me $e_{2}$-Cyclopentane & .04 & $\ldots$ & .02 & -.... & .01 & -.... & .01 & $\ldots$ & -.... & .18 & .12 & .14 & .03 & .19 & .01 & .02 \\
\hline cis-1,2-me 2 -Cyclopentane..... & .07 & .01 & .03 & -...- & .01 & -...- & .01 & -.... & $\ldots$ & .03 & .02 & .16 & .05 & .21 & .03 & .02 \\
\hline trans-1,2-me $e_{2}$-Cyclopentane & .06 & -.... & .05 & -.... & $\ldots$ & ..... & .02 & -.... & ..... & .02 & .02 & .14 & .04 & .21 & .03 & .02 \\
\hline cis-1,3-me 2 -Cyclopentane $\ldots$. & .07 & $\ldots$ & .04 & -..-. & .03 & -...- & .08 & -..- & .07 & .08 & .13 & .17 & .04 & .21 & .02 & .04 \\
\hline trans-1,3-me ${ }_{2}$-Cyclopentane & .07 & -.... & .04 & -.... & .04 & $\ldots$ & .09 & $-\ldots$ & .11 & .07 & .12 & .16 & .05 & .23 & .03 & .04 \\
\hline 1-Heptene........ & .10 & .... & - & $\ldots-$ & - & - & $-\ldots$ & - & , & -.... & $\ldots$. & .07 & .02 & .05 & -..... & -... \\
\hline 4,4-me 2 -1-Pentene ${ }^{2}$ & .04 & -...- & .02 & .03 & -...- & -...- & $-\ldots$ & $-\ldots$ & -.... & .10 & .16 & .02 & $-\ldots$ & .02 & $-\ldots$ & -..- \\
\hline 2,3,3-mes-1-Butene 2 - $\ldots$ & .05 & -...- & .02 & ...... & - & .... & - & - & -... & .11 & .10 & .05 & $\ldots$ & .04 & .... & - . \\
\hline
\end{tabular}

1 Also gives peaks $\mathrm{C}_{7} \mathrm{H}_{5}++0.02, \mathrm{C}_{7} \mathrm{H}_{11}{ }^{++} 0.01$.

${ }_{2}$ Also give small peaks at $\mathrm{C}_{6} \mathrm{H}_{11}{ }^{++}$ 
among the $\mathrm{C}_{5}$ ions is frequently much like that in the $\mathrm{C}_{5}$ hydrocarbons, though the intensity is less. Trans-3-hexene is an exception, for here $\mathrm{C}_{5} \mathrm{H}_{9}{ }^{++}$ is the largest peak, and this peak is small or absent in all other $\mathrm{C}_{6}$ hydrocarbons. The benzene spectrum is given in table 9 .

\section{6. $\mathrm{C}_{7}$ Hydrocarbons}

Table 6 lists doubly charged ions of $19 \mathrm{C}_{7}$ hydrocarbons. Ions $\mathrm{C}_{7} \mathrm{H}_{2}{ }^{++}$and $\mathrm{C}_{7} \mathrm{H}_{4}{ }^{++}$would be masked by singly charged ions in $\mathrm{C}_{7} \mathrm{H}_{16}$ and $\mathrm{C}_{7} \mathrm{H}_{14}$ isomers. The nine $\mathrm{C}_{7} \mathrm{H}_{16}$ isomers give a variety of mass spectra of doubly charged ions with $\mathrm{C}_{5}$ ions the most prominent, except in one case, 2,2-dimethylpentane, which has a maximum peak at $\mathrm{C}_{6} \mathrm{H}_{9}{ }^{++}$. The only other $\mathrm{C}_{7}$ with a maximum at this peak is 4,4-dimethyl-1-pentene. This is a structurally similar molecule, except that it contains a double bond. The cyclies all give quite similar doubly charged ion spectra with $\mathrm{C}_{7} \mathrm{H}_{8}{ }^{++}$the maximum peak. Table 9 gives the spectrum of methylbenzene.

\section{7. $\mathrm{C}_{8}$ Hydrocarbons}

Table 7 lists doubly charged ion spectra of $15 \mathrm{C}_{8} \mathrm{H}_{18}$ isomers, 22 cyclic and olefin $\mathrm{C}_{8} \mathrm{H}_{16}$

TABLE 7. Doubly charged ions of $\mathrm{C}_{8}$ hydrocarbons

Relative intensities in percentage of maximum peak in spectrum

\begin{tabular}{|c|c|c|c|c|c|c|c|c|c|c|c|c|c|c|c|c|c|}
\hline Compound & $\mathrm{C}_{5} \mathrm{H}_{2}$ & $\mathrm{C}_{5} \mathrm{H}_{4}$ & $\mathrm{C}_{5} \mathrm{H}_{6}$ & $\mathrm{C}_{5} \mathrm{H}_{8}$ & $\mathrm{C}_{5} \mathrm{H}_{10}$ & $\mathrm{C}_{6} \mathrm{H}_{7}$ & $\mathrm{C}_{6} \mathrm{H}_{9}$ & $\mathrm{C}_{6} \mathrm{H}_{11}$ & $\mathrm{C}_{7} \mathrm{H}_{5}$ & $\mathrm{C}_{7} \mathrm{H}_{6}$ & $\mathrm{C}_{7} \mathrm{H}_{7}$ & $\mathrm{C}_{7} \mathrm{H}_{3}$ & $\mathrm{C}_{7} \mathrm{H}_{9}$ & $\mathrm{C}_{7} \mathrm{H}_{10}$ & $\mathrm{C}_{7} \mathrm{H}_{11}$ & $\mathrm{C}_{7} \mathrm{H}_{12}$ & $\mathrm{C}_{7} \mathrm{H}_{13}$ \\
\hline \multicolumn{18}{|l|}{$n$-Octane. } \\
\hline 2-me-Heptane..... & 0.01 & 0.01 & -... & -..- & .... & .... & ...- & .... & -... & .... & .... & .06 & 0.01 & 0.04 & $\ldots$ & 0.03 & -... \\
\hline 3-me-Heptane ..... & .02 & .01 & $\ldots$ & $\ldots$ & - - - & $\ldots$ & $\ldots$ & -.... & $\ldots$ & $\ldots$ & -... & .03 & $\ldots$ & .01 & $\ldots$ & ..... & $\ldots$ \\
\hline 4-me-Heptane.... & .02 & .02 & $\ldots$ & $\ldots$ & $\ldots$ & ...- & .... & $-\ldots$ & -... & -... & $\ldots$ & .02 & -... & $\ldots$ & $\ldots$ & - n & - n \\
\hline 2,2-me -Hexane -.... & .... & $\ldots$ & $\ldots$ & .... & $\ldots$ & .... & ... & -...- & -... & 0.03 & $\ldots$ & .05 & $\ldots$ & .04 & $\ldots$ & .04 & -... \\
\hline 2,3-me $\mathrm{m}_{2}$-Hexane ..... & .01 & .01 & $\ldots$ & $\ldots$ & $\ldots$ & $\ldots$ & -..- & $\ldots$ & $\ldots$ & $\ldots$ & ...- & .03 & .... & $\ldots$ & ..... & -... & $\ldots$ \\
\hline 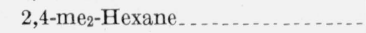 & .01 & $\ldots$ & -... & -... & $\ldots$ & -... & 0.01 & $\ldots$ & .... & -... & -... & .04 & $\ldots$ & .01 & $\ldots$ & $\ldots$ & $\ldots$ \\
\hline 2,5-me -Hexane $_{-} . . . .$. & -... & -... & $\ldots$ & $\ldots$ & $\ldots$ & $\ldots$ & .13 & 0.03 & .... & .... & -... & .08 & $\ldots$ & .05 & $\ldots$ & .05 & $\ldots$ \\
\hline 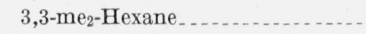 & .01 & .01 & $\ldots$ & ...- & ..... & -... & .... & $\ldots$ & .... & -..- & $-\ldots$ & .02 & $\ldots$ & -.... & -.... & $-\ldots$ & $\ldots$ \\
\hline 3,4-me 2 -Hexane $\ldots \ldots$ & .01 & .01 & $\ldots$ & -... & $\ldots$ & $\ldots$ & -... & $\ldots$ & -... & -... & $\ldots$ & .02 & $\ldots$ & $\ldots$ & -.... & $\ldots$ & $\ldots$ \\
\hline $2,2,3$-me entane $_{2}$. . . . . . . . . & .... & $\ldots$ & -... & -... & $\ldots$ & .... & -... & $\ldots$ & $\ldots$ & .03 & $\ldots$ & .03 & $\ldots$ & $\ldots$ & -.... & -.... & $\ldots$ \\
\hline 2,2,4-mes-Pentane $\ldots$ & .01 & .01 & -..- & $\ldots$ & -.... & 0.01 & .14 & .07 & $\ldots$ & .03 & $\ldots$ & .04 & $\ldots$ & .03 & & .03 & 0.01 \\
\hline $2,3,3-\mathrm{me}_{3}$-Pentane & .01 & -... & -... & .... & $\ldots$ & $\ldots$ & -... & $\ldots$ & .... & -..- & $\ldots$ & .03 & $\ldots$ & $\ldots$. & & $\ldots$ & $\ldots$ \\
\hline 2,3,4-mes-Pentane $\ldots . . .$. & .... & -... & -... & $\ldots$ & $\ldots$ & -... & .02 & ..... & -... & $\ldots$ & $\ldots$ & .04 & …- & $\ldots$ & & $\ldots$ & $\ldots$ \\
\hline 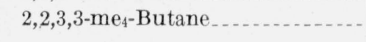 & -... & $-\ldots$ & $-\cdots$ & -... & $\ldots$ & -..- & .02 & $\ldots$ & $-\ldots$ & $: 03$ & -..- & .02 & -... & $-\ldots$ & & ..... & 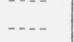 \\
\hline 1-Octene..... & -... & .... & $\ldots$ & -... & ..... & .... & -...- & ..... & $\ldots$ & $\ldots$ & $\ldots$ & .03 & -... & ..... & $\ldots$ & -.... & .... \\
\hline trans-4-Octene....... & -... & 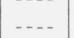 & $\ldots$ & -... & $\ldots$ & $\ldots$ & 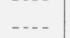 & ..... & $\ldots$ & 10 & $\ldots$ & 03 & -... & - n & $\ldots$ & n & - n \\
\hline 2,4,4-mes-1-Pentene. ... & $\ldots$ & -... & .... & $\ldots$ & ..... & .02 & -..- & $\ldots$ & ... & .07 & .04 & .21 & .04 & .08 & 0.03 & .01 & .30 \\
\hline 2,4,4-me $\mathrm{e}_{3}-2$-Pentene............ & -... & -... & $-\ldots$ & -... & $\ldots$ & .08 & -... & $\ldots$ & 0.02 & .29 & .11 & 68 & .12 & 17 & .10 & 01 & .22 \\
\hline \multicolumn{18}{|l|}{ Cyclohexanes: } \\
\hline $1,1-\mathrm{me}_{2} \ldots$ & -... & .... & $\ldots$ & $\ldots$ & $\ldots$ & .01 & $\ldots$ & $\ldots$ & $\ldots$ & .23 & .07 & .37 & .11 & .08 & .03 & .03 & .04 \\
\hline cis-1,2-me $2, \ldots$. & .... & -... & $\ldots$ & -... & $\ldots$ & -... & -... & $\ldots$ & -... & .21 & .05 & .25 & .04 & .04 & .01 & $\ldots$ & $\ldots$ \\
\hline trans $-1,2-\mathrm{me}_{2} \ldots$ & .... & .... & .... & $\ldots$ & ..... & .... & $\ldots$ & $\ldots$ & .... & .20 & .05 & .20 & .02 & .02 & ..... & -.... & $\ldots$ \\
\hline cis-1,4-men $\mathrm{e}_{2} \ldots \ldots \ldots$ & $\ldots$ & .... & $\ldots$ & .... & $\ldots$ & .02 & .03 & -.... & - & .21 & .06 & .26 & .05 & .05 & $\ldots$ & -... & -... \\
\hline trans $-1,4-m_{2} \ldots$. & .... & .... & .... & $\ldots$ & -.... & .01 & .02 & & .01 & .17 & .05 & .22 & .04 & .04 & .01 & .01 & .... \\
\hline cis-1,3-me $\mathrm{me}_{2} \ldots . .$. & .... & .... & $\ldots$ & -... & -.... & .02 & .01 & $\ldots$ & $\ldots$ & .14 & .08 & .14 & .05 & .05 & -.... & .02 & .... \\
\hline trans-1,3-me $2 \ldots$. & .... & .... & .... & $\ldots$ & $\ldots$ & .02 & .01 & $\ldots$ & $\ldots$ & .21 & .10 & .27 & .06 & .05 & $\ldots$ & -...- & -... \\
\hline 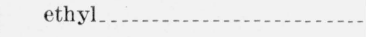 & .03 & .01 & 0.01 & -... & -.... & -... & $-\ldots$ & $-\ldots$ & $\cdots$ & .10 & .03 & .08 & .03 & .03 & $-\ldots$ & $-\ldots$ & $-\ldots$ \\
\hline \multicolumn{18}{|l|}{ Cyclopentanes: } \\
\hline n-Propyl... & .03 & .02 & -... & -... & , & .... & - ... & $\ldots$. & $\ldots$ & .11 & .04 & .11 & .01 & ...... & ..... & ..... & .... \\
\hline$i$-Propyl ...... & .02 & .01 & $\ldots$ & $\ldots$ & $\ldots$ & $\ldots$ & $-\cdots$ & -.... & -... & .20 & .09 & .30 & .07 & .04 & .01 & $\ldots$ & .... \\
\hline 1-me-1-eth .......... & .02 & .03 & $\ldots$ & $\ldots$ & ..... & $\ldots$ & $\ldots$ & ..... & .... & .13 & .05 & .13 & .02 & .01 & -...- & ...... & .02 \\
\hline cis-1-me-2-eth . . .......... & .02 & $-\ldots$ & $-\ldots$ & $-\ldots$ & $\ldots$ & $\ldots$ & $-\ldots$ & $\ldots$ & $-\ldots$ & 16 & .05 & .16 & .02 & .03 & - n & - & $-\ldots$ \\
\hline $1,1,2-m_{3} \ldots$ & .02 & .02 & -... & $\ldots$ & ..... & $\ldots$ & $\ldots$ & $\ldots$ & .... & .22 & .09 & .29 & .08 & .04 & .03 & ..... & ...- \\
\hline $1,1,3-m_{3}$ & .01 & .02 & .02 & $\ldots$ & & .05 & .04 & $\ldots$ & .02 & .30 & .15 & .53 & .16 & .10 & .09 & .04 & .24 \\
\hline cis, cis, cis-1,2,3-mes_........... & .01 & .01 & .01 & -... & ..... & $\ldots$ & $\ldots$ & ..... & -... & .12 & .05 & .16 & .02 & .02 & $\ldots$ & -.... & $\ldots$ \\
\hline cis, trans, cis-1,2,3-me $\mathrm{me}_{3} \ldots \ldots$ & .02 & .02 & .01 & $-\ldots$ & - . - - & $\ldots$ & -... & $\ldots$ & $\ldots$ & .16 & .07 & .22 & .03 & .03 & -.... & $\ldots$ & .... \\
\hline cis, trans, cis-1,2,4-me $\mathrm{m}_{3} \ldots \ldots$ & .01 & .01 & .01 & .01 & 0.04 & -...- & -... & $\ldots$ & $\ldots$ & .12 & .04 & .14 & .03 & -.... & -.... & -.... & $\ldots$ \\
\hline cis, cis, trans-1,2,4-me $\mathrm{m}_{3} \ldots \ldots$ & .01 & .01 & .91 & .01 & .04 & $\ldots$ & $-\cdots$ & $\ldots$ & -... & .09 & .05 & .13 & .03 & .01 & $\ldots$ & -...- & -... \\
\hline 4-Vinyl-1-cyclohexene 1..... & .04 & $-\cdots$ & -..- & -..- & -... & -... & -... & ..... & .03 & .25 & .29 & .11 & .10 & $\ldots$. & .... & $\ldots$ & $-\ldots$ \\
\hline
\end{tabular}

${ }_{1}$ Also gives peaks $\mathrm{C}_{7} \mathrm{H}_{2}++0.18, \mathrm{C}_{7} \mathrm{H}_{3}++0.02, \mathrm{C}_{7} \mathrm{H}_{4}++0.07, \mathrm{C}_{8} \mathrm{H}_{3}{ }^{++} 0.04, \mathrm{C}_{8} \mathrm{H}_{5}{ }^{++} 0.01, \mathrm{C}_{8} \mathrm{H}_{7}++0.09, \mathrm{C}_{8} \mathrm{H}_{9}{ }^{++} 0.20, \mathrm{C}_{8} \mathrm{H}_{11}++0.01$. 
TABLE 8. Doubly charged ions of $\mathrm{C}_{9}$ hydrocarbons

Relative intensities in percentage of maximum peak in spectrum

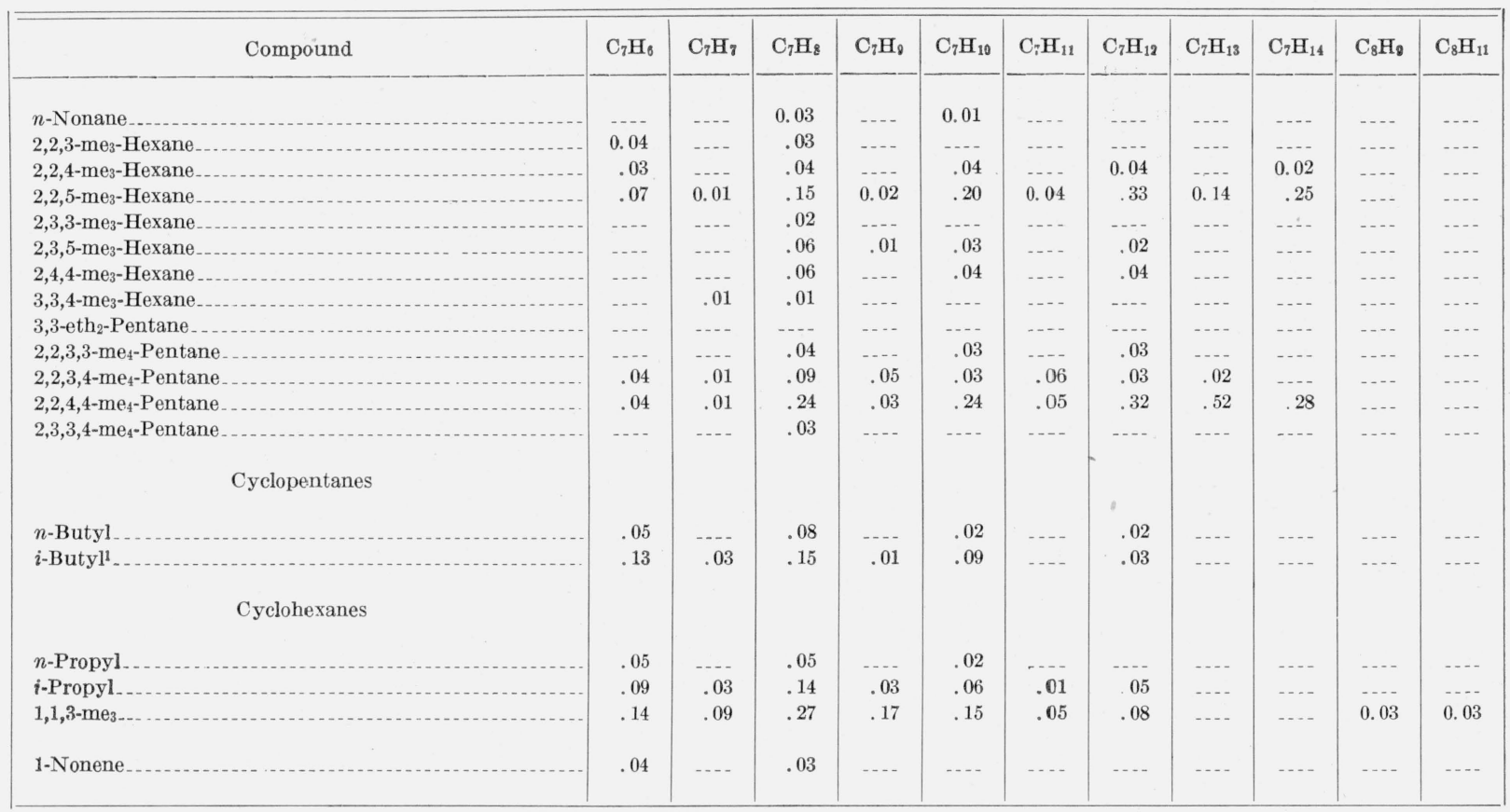

1 This also has a peak of 0.02 at $\mathrm{C}_{5} \mathrm{H}_{2}++$.

isomers, and $1 \mathrm{C}_{8} \mathrm{H}_{12}$ molecule. $\mathrm{C}_{8}$ ions with an even number of hydrogen atoms would be masked by singly charged ions. Three $\mathrm{C}_{8} \mathrm{H}_{18}$ isomers not included in the table give no observable doubly charged ions. These are the three octanes that have ethyl side chains; 3-ethylhexane, 2-methyl-3ethylpentane and 3-methyl-3-ethylpentane. Most of the $\mathrm{C}_{8} \mathrm{H}_{18}$ molecules give small doubly charged peaks, and in 12 cases the maximum peak is $\mathrm{C}_{7} \mathrm{H}_{8}{ }^{++}$. The largest peaks and the greatest number of peaks are in 2,2,4-trimethylpentane. Among the $\mathrm{C}_{8} \mathrm{H}_{16}$ cyclics, the most abundant doubly charged ions are in 1,1,3-trimethylcyclopentane and of the four olefins 2,4,4-trimethyl-2pentene has the largest peaks. These three compounds have a structural similarity that is partially concealed by the rules used for numbering the side chains. The $\mathrm{C}_{8} \mathrm{H}_{12}$ molecule, 4-ethenyl-1cyclohexene, shows many peaks not found in any other molecules in this table. The occurrence of $\mathrm{C}_{8}$ ions ranging from $\mathrm{C}_{8} \mathrm{H}_{3}{ }^{++}$to $\mathrm{C}_{8} \mathrm{H}_{11}{ }^{++}$is significant. The ions $\mathrm{C}_{7} \mathrm{H}_{2}{ }^{++}$and $\mathrm{C}_{7} \mathrm{H}_{4}{ }^{++}$would be masked in most of the other compounds in table 7 . The spectrum resembles somewhat the alkyl benzenes of table 9 .

\section{8. $\mathrm{C}_{9}$ Hydrocarbons}

Table 8 lists doubly charged ions of $13 \mathrm{C}_{9} \mathrm{H}_{20}$ isomers and six $\mathrm{C}_{9} \mathrm{H}_{18}$ isomers (cyclics and one olefin). One nonane, 3,3-diethylpentane, shows no doubly charged ions. In the octanes it was also found that isomers with ethyl side chains give no doubly charged peaks. With one exception the doubly charged ions of these molecules are $\mathrm{C}_{7}$ ions. The exception is 1,1,3-trimethylcyclohexane with two small peaks at $\mathrm{C}_{8} \mathrm{H}_{9}{ }^{++}$and $\mathrm{C}_{8} \mathrm{H}_{11}{ }^{++}$. This molecule gives the most intense doubly charged spectrum of the five cyclics listed. It was found that 1,1,3-trimethylcyclopentane gave the largest peaks of any of the $\mathrm{C}_{8} \mathrm{H}_{16}$ cyclics listed in table 7. A following section gives evidence that peaks at 96,97 , and 98 are $\mathrm{C}_{7} \mathrm{H}_{12}{ }^{++}, \mathrm{C}_{7} \mathrm{H}_{13}{ }^{++}$, and $\mathrm{C}_{7} \mathrm{H}_{14}{ }^{++}$; and not $\mathrm{C}_{8}{ }^{++}, \mathrm{C}_{8} \mathrm{H}^{++}$, and $\mathrm{C}_{8} \mathrm{H}_{2}{ }^{++}$.

It is of interest that $n$-butyl-cyclopentane and $n$-propylcyclohexane have similar spectra and that the iso-butyl and iso-propyl are also similar to each other and different from the other cyclics.

In general the doubly charged spectra of $\mathrm{C}_{9}$ hydrocarbons are much like those of $\mathrm{C}_{8}$ hydrocarbons. $\mathrm{C}_{7} \mathrm{H}_{8}{ }^{++}$is the maximum peak in most cases with only two striking exceptions. 
Relative intensities in percentage of maximum peak in spectrum

\begin{tabular}{|c|c|c|c|c|c|c|c|c|c|c|c|c|c|c|c|c|c|}
\hline Compound & $\mathrm{C}_{6} \mathrm{H}$ & $\mathrm{C}_{6} \mathrm{H}_{3}$ & $\mathrm{C}_{6} \mathrm{H}_{5}$ & $\mathrm{C}_{6} \mathrm{H}_{6}$ & $\mathrm{C}_{7} \mathrm{H}$ & $\mathrm{C}_{7} \mathrm{H}_{2}$ & $\mathrm{C}_{7} \mathrm{H}_{3}$ & $\mathrm{C}_{7} \mathrm{H}_{4}$ & $\mathrm{C}_{7} \mathrm{H}_{5}$ & $\mathrm{C}_{7} \mathrm{H}_{6}$ & $\mathrm{C}_{7} \mathrm{H}_{7}$ & $\mathrm{C}_{7} \mathrm{H}_{8}$ & $\mathrm{C}_{8} \mathrm{H}_{3}$ & $\mathrm{C}_{8} \mathrm{H}_{5}$ & $\mathrm{C}_{8} \mathrm{H}_{7}$ & $\mathrm{C}_{8} \mathrm{H}_{8}$ & $\mathrm{C}_{8} \mathrm{H}_{10}$ \\
\hline Benzene ${ }^{1} \ldots$ & 0.05 & 1.15 & 0.35 & 23.0 & $\ldots$ & -... & -.... & -.... & $\ldots$ & ..... & ..... & $\ldots$ & $-\ldots$ & $\ldots$ & - & - & 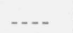 \\
\hline Me-Benzene ${ }^{3} \ldots \ldots$ & -... & .04 & $\ldots$ & $\ldots-$ & 0.13 & 1.73 & 0.42 & 0.97 & 0.49 & 4.87 & 2.35 & 3.45 & $\ldots$ & $\ldots$ & $-\ldots$ & $\ldots$ & - n \\
\hline Eth-Benzene & $\ldots$ & .04 & .02 & .... & $\ldots-$ & .10 & .02 & .05 & .05 & .05 & .20 & .02 & 0.05 & 0.08 & 0.32 & 0.24 & --.-. \\
\hline 1,2-me -benzene $_{2}$ & $\ldots$ & .04 & $\ldots$ & $\ldots$ & $\ldots$ & .15 & .05 & .08 & .05 & .05 & .28 & .03 & .06 & .11 & .73 & .66 & 10.2 \\
\hline 1,3-mè2-benzene & $-\ldots$ & .03 & .01 & ....- & $\ldots$ & .16 & .05 & .07 & .05 & .04 & .21 & .01 & .10 & .12 & .78 & .77 & 4.2 \\
\hline 1,4-me - benzene & $\ldots$ & -... & $\ldots$ & $\ldots$ & $\ldots$ & .06 & $\ldots$ & .15 & .04 & .02 & .22 & .02 & $-\ldots$ & .07 & .61 & .52 & ${ }^{4} .5$ \\
\hline
\end{tabular}

${ }^{1}$ Also has peaks at $\mathrm{C}_{4} \mathrm{H}_{3}++0.03$ and $\mathrm{C}_{5} \mathrm{H}_{2}++0.05$.

${ }^{2}$ Based on a peak of 0.20 at 39.5 from $\mathrm{C}^{13} \mathrm{C}_{5}{ }^{12} \mathrm{H}_{6}++$.

\section{Benzene and Alkyl Benzenes}

Table 9 gives doubly charged ions of some aromatics. These have been listed in a separate table for convenience of tabulation, because they include many ions not found in other hydrocarbons. The peak heights are larger than in other $\mathrm{C}_{8}, \mathrm{C}_{7}$, and $\mathrm{C}_{8}$ hydrocarbons.

Benzene has a very large $\mathrm{C}_{6} \mathrm{H}_{6}{ }^{++}$peak and only one small $\mathrm{C}_{5}$ peak in contrast to most of the spectra in table 5. Cyclohexene is the only compound in table 5 that has large $\mathrm{C}_{6}$ peaks.

Methylbenzene resembles qualitatively the $\mathrm{C}_{7} \mathrm{H}_{14}$ cyclics (table 6) except for differences definitely related to the fact that there are $8 \mathrm{H}$ atoms instead of $14 . \mathrm{C}_{7} \mathrm{H}_{2}^{++}$and $\mathrm{C}_{7} \mathrm{H}_{4}^{++}$would be masked by singly charged peaks in the cyclics.

The $\mathrm{C}_{8} \mathrm{H}_{10}$ aromatics give spectra quite unlike any of the $\mathrm{C}_{8}$ molecules of table 7 except the $\mathrm{C}_{8} \mathrm{H}_{12}$ molecule, 4-ethenyl-1-cyclohexene. It differs from this in having $\mathrm{C}_{8}$ ion peaks that are larger than the $\mathrm{C}_{7}$ peaks. The estimate of $\mathrm{C}_{8} \mathrm{H}_{10}{ }^{++}$ is based on very small isotope peaks and is quite uncertain. The fact that $\mathrm{C}_{7} \mathrm{H}_{8}{ }^{++}$is small or missing here in contrast to other $\mathrm{C}_{8}$ molecules undoubtedly is a consequence of the fact that dissociation by removal of $\mathrm{CH}_{3}$ gives $\mathrm{C}_{7} \mathrm{H}_{7}++$, whereas multiple dissociation or rearrangement is required to give $\mathrm{C}_{7} \mathrm{H}_{8}{ }^{++}$.

\section{Hydrocarbons with 10 or More Carbon Atoms}

There is little published data on these heavier hydrocarbons. $n$-Decane, $\mathrm{C}_{10} \mathrm{H}_{22}$, gives only two small peaks, $\mathrm{C}_{5} \mathrm{H}_{2}{ }^{++} 0.02$ and $\mathrm{C}_{5} \mathrm{H}_{4}{ }^{++} 0.02$.

1-Decene, $\mathrm{C}_{10} \mathrm{H}_{20}$, shows only one doubly charged ion, $\mathrm{C}_{7} \mathrm{H}_{8}{ }^{++} 0.03$. This is also the only ion in 1octene, whereas l-nonene gave this ion and $\mathrm{C}_{7} \mathrm{H}_{6}{ }^{++}$.
${ }^{3}$ Also has peaks at $\mathrm{C}_{5} \mathrm{H}_{2}{ }^{++} 0.17, \mathrm{C}_{5} \mathrm{H}_{3}{ }^{++} 0.02$, and $\mathrm{C}_{5} \mathrm{H}_{4}++0.11$.

${ }^{4}$ Based on a peak at 53.5 from $\mathrm{C}^{13} \mathrm{C}_{7}^{12} \mathrm{H}_{10}{ }^{++}$.

The four butylcyclohexanes give doubly charged spectra like the $\mathrm{C}_{9} \mathrm{H}_{18}$ cyclics, except that the peaks are smaller. All the ions have seven carbon atoms.

The Atlantic Refining Co. has published mass spectra [7] of the three diisopropylbenzenes $\left(\mathrm{C}_{12} \mathrm{H}_{18}\right)$ at an ionizing voltage of 50 volts. These include the heaviest doubly charged ions recorded to date. The three spectra are similar, and 1,3diisopropylbenzene gives the following peaks: $\mathrm{C}_{9} \mathrm{H}_{7}{ }^{++} 0.37, \mathrm{C}_{9} \mathrm{H}_{9}{ }^{++} 0.08, \mathrm{C}_{9} \mathrm{H}_{11}{ }^{++} 0.05, \mathrm{C}_{10} \mathrm{H}_{7}{ }^{++} 0.75$, $\mathrm{C}_{10} \mathrm{H}_{9}{ }^{++} 1.25, \quad \mathrm{C}_{10} \mathrm{H}_{11}{ }^{++} 2.79, \quad \mathrm{C}_{10} \mathrm{H}_{13}{ }^{++} 0.53, \quad$ and $\mathrm{C}_{11} \mathrm{H}_{15}{ }^{++} 0.65$.

\section{Ratio of Peaks at 70- and 50-Volt Ionizing Voltage}

The API tables gives mass spectra obtained with ionizing voltages of 50 and 70 volts and, in general, the doubly charged peaks are considerably larger at the higher voltage. The value of the ratio of the currents at 70 and 50 volts, $i(70) / i(50)$, is of interest, as it gives at least qualitative evidence as to the appearance potential of the ion.

A plot of ion current as a function of ionizing voltage rises at first almost linearly from the appearance potential but gradually becomes concave to the axis of abscissas approaching a flat maximum value at roughly 30 volts above the threshold. Ionizing voltages of 50 and 70 volts fall near the flat maximum for the more probable single ionization processes, and the ratio $i(70) / i(50)$ is nearly unity. However, if the appearance potential is high, then the ratio will be much greater than unity and approach infinity as the appearance potential approaches 50 volts.

There are a few direct measurements of appearance potentials of doubly charged ions and these 
are correlated with the observed values of $i(70) /$ $i(50)$ in table 10 . There is a rather large experimental uncertainty in the ratio amounting to at least 10 percent, and one can only conclude that a value of 1.2 to 1.3 corresponds to a potential between 30 and 36 volts and 1.4 to roughly 40 volts. Above 40 volts the ratio will increase rapidly with increasing appearance potential.

TABLE 10. Correlation of appearance potential and the ration of ion currents at 70 and 50 volts

\begin{tabular}{|c|c|c|c|c|}
\hline Compound & Reference & Ion & $\begin{array}{c}\text { Appearance } \\
\text { potential }\end{array}$ & $i(70) / i(50)$ \\
\hline $\mathrm{C}_{2} \mathrm{H}_{6 \ldots \ldots}$ & {$[2]$} & $\mathrm{C}_{2} \mathrm{H}_{5}++$ & 32 & 1. 25 \\
\hline $\mathrm{C}_{2} \mathrm{H}_{4 \ldots}$ & [3] & $\mathrm{C}_{2} \mathrm{H}_{3}{ }^{++}$ & 36 & 1.3 \\
\hline $\mathrm{C}_{6} \mathrm{H}_{6 \ldots}$ & [4] & $\mathrm{C}_{6} \mathrm{H}_{6}++$ & 27 & 1.0 \\
\hline $\mathrm{C}_{6} \mathrm{H}_{6} \ldots \ldots$ & $\ldots$ & $\mathrm{C}_{6} \mathrm{H}_{5}++$ & 30.4 & 1. 2 \\
\hline $\mathrm{C}_{6} \mathrm{H}_{6} \ldots$ & .... & $\mathrm{C}_{6} \mathrm{H}_{3}++$ & 39.8 & 1.4 \\
\hline
\end{tabular}

Observed values of the ratio $i(70) / i(50)$ for the more abundant doubly charged ions in hydrocarbons with two to five carbon atoms and for benzene are listed in table 11 . It is found that the ratios for the same ions in different isomers are nearly equal. In general, the range of values indicated is no greater than the experimental uncertainty. This is true even when the isomers are chemically different, as in the case of cyclics and olefins. The table is arranged to show that the ratio increases as the number of hydrogen atoms removed increases, and that ionization processes involving removal of equal numbers of hydrogen atoms from different molecules give the same ratio within the range of experimental uncertainty. There is not a progressive decrease in the ratio as the number of $H$ atoms removed becomes less, but the ratio remains nearly the same for removing $7 \mathrm{H}$ and $8 \mathrm{H}$ and similarly for $5 \mathrm{H}$ and $6 \mathrm{H}, 3 \mathrm{H}$, and $4 \mathrm{H}$ and for $1 \mathrm{H}$ and $2 \mathrm{H}$.

Interpretation of ratios in terms of appearance potentials.-The interpretation of this is fairly obvious. Studies of appearance potentials of singly charged ions show that frequently but not always, the hydrogen atoms are removed in pairs to form $\mathrm{H}_{2}$. Thus Delfosse and Bleakney [6] find that in propane, $\mathrm{C}_{3} \mathrm{H}_{5}{ }^{+}$is produced by the transition $\mathrm{C}_{3} \mathrm{H}_{8} \rightarrow \mathrm{C}_{3} \mathrm{H}_{5}{ }^{+}+\mathrm{H}_{2}+\mathrm{H}$ requiring 14 volts. The process $\mathrm{C}_{3} \mathrm{H}_{8} \rightarrow \mathrm{C}_{3} \mathrm{H}_{4}{ }^{+}+2 \mathrm{H}_{2}$ occurs at 14.7 volts, and $\mathrm{C}_{3} \mathrm{H}_{8} \rightarrow \mathrm{C}_{3} \mathrm{H}_{3}{ }^{+}+2 \mathrm{H}_{2}+\mathrm{H}$ requires 15.7 volts. As it requires about three electron volts
TABLE 11.-Ratio of peaks at $\% 0$ - and 50-volt ionizing voltage

\begin{tabular}{|c|c|c|}
\hline Ionization process & $i(70) / i(50)$ & Remarks \\
\hline $\mathrm{C}_{5} \mathrm{H}_{12} \rightarrow \mathrm{C}_{5} \mathrm{H}_{2}{ }^{++}+10 \mathrm{H}_{\ldots} .$. & 4.5 to 6.8 & 3 isomers. \\
\hline $\mathrm{C}_{5} \mathrm{H}_{10} \rightarrow \mathrm{C}_{5} \mathrm{H}_{2}+++8 \mathrm{H}_{\ldots} \ldots$ & 2.8 to 3.7 & 7 isomers, mean 3.5 . \\
\hline $\mathrm{C}_{4} \mathrm{H}_{10} \rightarrow \mathrm{C}_{4} \mathrm{H}_{3}+++7 \mathrm{H}_{\ldots} \ldots$ & 2.2 and 3.5 & 2 isomers. \\
\hline $\mathrm{C}_{3} \mathrm{H}_{8} \rightarrow \mathrm{C}_{3} \mathrm{H}_{2}{ }^{++}+6 \mathrm{H} \ldots$ & 2.0 & Propane. \\
\hline $\mathrm{C}_{5} \mathrm{H}_{8} \rightarrow \mathrm{C}_{5} \mathrm{H}_{2}+++6 \mathrm{H}_{-}$ & 2.2 and 2.45 & 2 isomers. \\
\hline $\mathrm{C}_{5} \mathrm{H}_{10} \rightarrow \mathrm{C}_{5} \mathrm{H}_{4}+++6 \mathrm{H}$ & 1.6 to 1.9 & 7 isomers, mean 1.7 . \\
\hline $\mathrm{C}_{3} \mathrm{H}_{8} \rightarrow \mathrm{C}_{3} \mathrm{H}_{3}+++5 \mathrm{H}_{--}$. & 1.9 & Propane. \\
\hline $\mathrm{C}_{4} \mathrm{H}_{8} \rightarrow \mathrm{C}_{4} \mathrm{H}_{3}{ }^{++}+5 \mathrm{H}_{-}$ & 1.85 to 1.88 & 4 isomers. \\
\hline $\mathrm{C}_{4} \mathrm{H}_{10} \rightarrow \mathrm{C}_{4} \mathrm{H}_{5}{ }^{++}+5 \mathrm{H}_{-}$. & 2.0 & 2 isomers. \\
\hline $\mathrm{C}_{5} \mathrm{H}_{8} \rightarrow \mathrm{C}_{5} \mathrm{H}_{3}+++5 \mathrm{H}_{\ldots}$ & 1.7 and 1.9 & 2 isomers. \\
\hline $\mathrm{C}_{3} \mathrm{H}_{6} \rightarrow \mathrm{C}_{3} \mathrm{H}_{2}+++4 \mathrm{H} \ldots$ & 1.43 and 1.48 & 2 isomers. \\
\hline $\mathrm{C}_{3} \mathrm{H}_{8} \rightarrow \mathrm{C}_{3} \mathrm{H}_{4}+++4 \mathrm{H}_{-.}$ & 1.5 & Propane. \\
\hline $\mathrm{C}_{5} \mathrm{H}_{8} \rightarrow \mathrm{C}_{5} \mathrm{H}_{4}^{++}+4 \mathrm{H}_{\ldots} \ldots$ & 1.3 and 1.4 & 2 isomers. \\
\hline $\mathrm{C}_{5} \mathrm{H}_{10} \rightarrow \mathrm{C}_{5} \mathrm{H}_{6}+++4 \mathrm{H} \ldots$ & 1.1 to 1.5 & 7 isomers, mean 1.2 . \\
\hline $\mathrm{C}_{3} \mathrm{H}_{6} \rightarrow \mathrm{C}_{3} \mathrm{H}_{3}{ }^{++}+3 \mathrm{H}_{-}$ & 1.4 & 2 isomers. \\
\hline $\mathrm{C}_{4} \mathrm{H}_{6} \rightarrow \mathrm{C}_{4} \mathrm{H}_{3}+++3 \mathrm{H}_{-}$ & 1.42 to 1.46 & 4 isomers, mean 1.44. \\
\hline $\mathrm{C}_{5} \mathrm{H}_{8} \rightarrow \mathrm{C}_{5} \mathrm{H}_{5}^{++}+3 \mathrm{H}_{-.}$ & 1.2 and 1.35 & 2 isomers. \\
\hline $\mathrm{C}_{6} \mathrm{H}_{6} \rightarrow \mathrm{C}_{6} \mathrm{H}_{3}+++3 \mathrm{H}_{\ldots}$ & 1.4 & $\begin{array}{c}\text { Benzene, appearance } \\
\text { potential } 39.8 \text { volts. }\end{array}$ \\
\hline $\mathrm{C}_{3} \mathrm{H}_{6} \rightarrow \mathrm{C}_{3} \mathrm{H}_{4}^{++}+2 \mathrm{H}_{-}$ & 1.3 & 2 isomers. \\
\hline $\mathrm{C}_{5} \mathrm{H}_{8} \rightarrow \mathrm{C}_{5} \mathrm{H}_{6}+++2 \mathrm{H}_{-.}$ & 1.0 and 1.2 & 2 isomers. \\
\hline $\mathrm{C}_{5} \mathrm{H}_{10} \rightarrow \mathrm{C}_{5} \mathrm{H}_{8}++2 \mathrm{H} \ldots$ & 1.1 to 1.3 & 3 isomers, mean 1.2 . \\
\hline $\mathrm{C}_{2} \mathrm{H}_{4} \rightarrow \mathrm{C}_{2} \mathrm{H}_{3}{ }^{++}+\mathrm{H}_{\ldots}$. & 1.3 & $\begin{array}{l}\text { Ethylene, appearance, } \\
\text { potential } 36 \text { volts. }\end{array}$ \\
\hline $\mathrm{C}_{2} \mathrm{H}_{6} \rightarrow \mathrm{C}_{2} \mathrm{H}_{5}^{++}+\mathrm{H}_{\ldots}$. & 1.25 & $\begin{array}{l}\text { Ethane,appearance, poten- } \\
\text { tial } 32 \text { volts. }\end{array}$ \\
\hline $\mathrm{C}_{3} \mathrm{H}_{6} \rightarrow \mathrm{C}_{3} \mathrm{H}_{5}^{++}+\mathrm{H}_{-.}$. & 1.3 and 1.2 & 2 isomers. \\
\hline $\mathrm{C}_{4} \mathrm{H}_{6} \rightarrow \mathrm{C}_{4} \mathrm{H}_{5}+++\mathrm{H}_{\ldots}$. & 1.2 to 1.4 & 4 isomers, mean 1.3 . \\
\hline $\mathrm{C}_{5} \mathrm{H}_{8} \rightarrow \mathrm{C}_{5} \mathrm{H}_{7}+++\mathrm{H}_{\ldots}$ & 1.1 and 1.25 & 2 isomers. \\
\hline $\mathrm{C}_{6} \mathrm{H}_{6} \rightarrow \mathrm{C}_{6} \mathrm{H}_{5}+++\mathrm{H}_{-}$ & 1.2 & $\begin{array}{l}\text { Benzene, appearance po- } \\
\text { tential } 30.4 \text { volts. }\end{array}$ \\
\hline $\mathrm{C}_{5} \mathrm{H}_{8} \rightarrow \mathrm{C}_{5} \mathrm{H}_{8}++\ldots$ & 1.1 & Isoprene. \\
\hline $\mathrm{C}_{6} \mathrm{H}_{6} \rightarrow \mathrm{C}_{6} \mathrm{H}_{6}++\ldots$ & 1.0 & $\begin{array}{l}\text { Benzene, appearance po- } \\
\text { tential } 27 \text { volts. }\end{array}$ \\
\hline
\end{tabular}

to remove each $\mathrm{H}$ atom from a hydrocarbon and the energy of recombination $2 \mathrm{H} \rightarrow \mathrm{H}_{2}$ is 4.5 electron volts, it makes a large difference whether the pairs are removed as $\mathrm{H}_{2}$ or $2 \mathrm{H}$. As the different radicals have different ionization potentials and may have excess kinetic energy, the appearance potentials cannot be computed from these considerations alone. However, the results of table 11 would indicate that dissociation into $\mathrm{H}_{2}$ usually occurs in double-ionization processes. That is, it requires about the same work to remove $4 \mathrm{H}_{2}$ and $3 \mathrm{H}_{2}+\mathrm{H}, 3 \mathrm{H}_{2}$ and $2 \mathrm{H}_{2}+\mathrm{H}, 2 \mathrm{H}_{2}$ and $\mathrm{H}_{2}+\mathrm{H}$.

From comparison with the few instances in which the appearance potential is known, it appears that all double ionization processes involving removal of $\mathrm{H}$ or $\mathrm{H}_{2}$ require $30^{\circ}$ to 36 volts. Processes involving removal of $\mathrm{H}_{2}+\mathrm{H}$ or $2 \mathrm{H}_{2}$ require 
roughly 40 volts. Processes involving removal of $2 \mathrm{H}_{2}+\mathrm{H}$ and $3 \mathrm{H}_{2}$ must fall in a narrow voltage range above 40 volts, whereas removal of $5 \mathrm{H}_{2}$ must require nearly 50 volts.

Molecules with more than five carbon atoms give values of $i(70) / i(50)$ somewhat less than those listed in table 11 for removal of a given number of $\mathrm{H}$ atoms. Thus in seven $\mathrm{C}_{7} \mathrm{H}_{14}$ cyclics in the process $\mathrm{C}_{7} \mathrm{H}_{14} \rightarrow \mathrm{C}_{7} \mathrm{H}_{6}{ }^{++}+8 \mathrm{H}$, the ratio ranges from 1.3 to 1.5 as compared with 3.5 given in table 11. In the same isomers $\mathrm{C}_{7} \mathrm{H}_{14} \rightarrow \mathrm{C}_{7} \mathrm{H}_{8}{ }^{++}+6 \mathrm{H}$ gives a mean ratio of 1.2 , as compared with the mean 1.8 for the values in table 11 for loss of $6 \mathrm{H}$.

In table 7 three of the $\mathrm{C}_{8} \mathrm{H}_{16}$ isomers give large doubly charged peaks of mass 97 . This could be either $\mathrm{C}_{8} \mathrm{H}^{++}$or $\mathrm{C}_{7} \mathrm{H}_{13}{ }^{++}$. The ratio $i(70) / i(50)$ is 1.15 to 1.17 , indicating a low appearance potential, and for this reason the ion has been ascribed to $\mathrm{C}_{7} \mathrm{H}_{13}{ }^{++}$. Removing $15 \mathrm{H}$ atoms would require a very high appearance potential. Similarly 2,2,4,4-tetramethylpentane in table 8 gives ions of masses 96,97 , and 98 . The ratios are in the range 1.13 to 1.18 , and the ions have been ascribed to $\mathrm{C}_{7} \mathrm{H}_{12}{ }^{++}, \mathrm{C}_{7} \mathrm{H}_{13}{ }^{++}$, and $\mathrm{C}_{7} \mathrm{H}_{14}{ }^{++}$.

\section{Summary and Discussion}

The doubly charged ion spectra of hydrocarbons with three, four, or five carbon atoms follow some simple rules that can be summarized as follows:

1. In the most probable ionization processes carbon bonds are not broken, but all except two, three, or four hydrogen atoms are removed.

2 . The relative intensity distribution is similar in all hydrocarbons with the same number of carbon atoms.

3. The magnitude of the peaks is least for saturated molecules, greater for monoolefins and alkylcyclics and greatest for diolefins and cycloolefins.

4. Ion peaks tend to alternate in intensity with larger values for ions with an even number of $\mathrm{H}$ atoms.

These rules are in contrast to the case of singly charged ions. For these the most probable ionization processes frequently involve breaking carbon bonds, the spectra depend considerably on the structure of the molecule and are quite different for paraffins, olefins, and diolefins. Ions with an odd number of $\mathrm{H}$ atoms tend to be more abundant.
Doubly charged ion spectra of molecules with six or more carbon atoms are quite different from the lighter hydrocarbons. The most probable ionization processes involve breaking of carbon bonds in all these except the alkylbenzenes and the $\mathrm{C}_{7}$ cyclics. Structural differences and the degree of saturation give large differences in the spectra. $\mathrm{C}_{5} \mathrm{H}_{2}{ }^{++}$is often the maximum peak in $\mathrm{C}_{6}$ hydrocarbons and in $\mathrm{C}_{7}$ paraffins. In other $\mathrm{C}_{7}$ hydrocarbons and in $\mathrm{C}_{8}$ and $\mathrm{C}_{9}$ hydrocarbons, $\mathrm{C}_{7} \mathrm{H}_{8}{ }^{++}$is often the maximum peak. Rules 3 and 4 remain true, in general, with minor exceptions.

A qualitative consideration of the ionization process gives a partial explanation of some of the properties of doubly charged ion spectra. The least potential required to doubly ionize a large molecule would involve removal of two electrons from two atoms near each end of the molecule. (The Coulomb law of attraction makes the work required to remove the second electron least if it is removed from an atom as far away as possible from the first ion). The resulting ion with a charge near each end is subject to large disruptive forces, and probably a frequent result of double ionization is that the molecule ion dissociates into two singly charged ions. In general this process will be indistinguishable from single ionization processes, but Stevenson and Hipple [8] point out that the appearance potential of $\mathrm{CH}_{3}{ }^{+}$in the two butane isomers indicates that it comes from the process

$$
\mathrm{C}_{4} \mathrm{H}_{10} \rightarrow \mathrm{C}_{3} \mathrm{H}_{7}{ }^{+}+\mathrm{CH}_{3}{ }^{+}+2 e .
$$

If, however, a molecule loses most of its hydrogen atoms in the dissociation process, then the bonds between carbon atoms become double bonds and the bonding force is greatly increased. Evidently it is then sufficient to overcome the disruptive force of the double charge.

The intensity rules for doubly charged ions in molecules with three to five carbon atoms can be explained on the hypotheses that all but two to four $\mathrm{H}$ atoms must be removed to give stable ions, and that the probability of this ionization process decreases as the appearance potential increases. With unsaturated molecules and cyclics the number of $\mathrm{H}$ atoms to be removed is less, and the intensity increases. As hydrogen atoms tend to be removed in pairs to give $\mathrm{H}_{2}$, ions with an even number of $\mathrm{H}$ atoms are commonly more abundant than ions with an odd number. 
From table 11 it is seen that removing $10 \mathrm{H}$ atoms from $\mathrm{C}_{5} \mathrm{H}_{12}$ to give $\mathrm{C}_{5} \mathrm{H}_{2}{ }^{++}$requires nearly 50 volts. One reason why different ionization processes are involved in molecules with six or more carbon atoms is because the work required to remove all but two $\mathrm{H}$ atoms exceeds the ionizing voltage. In the $\mathrm{C}_{6} \mathrm{H}_{14}$ isomers, $\mathrm{C}_{5} \mathrm{H}_{2}{ }^{++}$is again the largest peak. This can be obtained by the least energy by removing $\mathrm{CH}_{4}$ and four $\mathrm{H}_{2}$ molecules. The fact that $\mathrm{C}_{5} \mathrm{H}_{2}^{++}, \mathrm{C}_{5} \mathrm{H}_{4}{ }^{++}$, and $\mathrm{C}_{5} \mathrm{H}_{6}{ }^{++}$are larger than the peaks with an odd number of $\mathrm{H}$ atoms, supports the view that loss of $\mathrm{CH}_{4}$ and $\mathrm{H}_{2}$ molecules actually occurs. In singly charged ion spectra, peaks with an odd number of $\mathrm{H}$ atoms are larger, and it is known that the more probable ionization processes involve dissociation with loss of $\mathrm{CH}_{3}$ and other radicals with an odd number of $\mathrm{H}$ atoms. (Ionization with dissociation into $\mathrm{CH}_{4}$ is known to occur but is less probable [8]).

In $\mathrm{C}_{6}$ hydrocarbons doubly charged ions with six carbon atoms are found in $\mathrm{C}_{6} \mathrm{H}_{12}$ isomers, and these ions are the most abundant ions in cyclohexene, $\mathrm{C}_{6} \mathrm{H}_{10}$, and in benzene, $\mathrm{C}_{6} \mathrm{H}_{6}$. $\mathrm{C}_{6}$ ions become more abundant as the number of $\mathrm{H}$ atoms decreases. This is also true of $\mathrm{C}_{7}$ ions in the $\mathrm{C}_{7}$ hydrocarbons. In $\mathrm{C}_{8}$ hydrocarbons there are no doubly charged $\mathrm{C}_{8}$ ions in $\mathrm{C}_{8} \mathrm{H}_{18}$ and $\mathrm{C}_{8} \mathrm{H}_{16}$ isomers. They are strong in 4-ethenyl-1-cyclohexene, $\mathrm{C}_{8} \mathrm{H}_{12}$, and predominant in the $\mathrm{C}_{8} \mathrm{H}_{10}$ alkylbenzenes.

In hydrocarbons with seven or more carbon atoms the ion $\mathrm{C}_{7} \mathrm{H}_{8}{ }^{++}$is commonly the most abundant ion. The empirical fact that such an ion is stable is another factor that makes the doubly charged ions of the heavier hydrocarbons differ from the lighter ones. Also in the heavier hydrocarbons certain configurations of carbon atoms seem to favor doubly charged ions such as the 2,2,4-trimethyl arrangement in $\mathrm{C}_{8}$ hydro- carbons. Other configurations, such as ethyl side chains in the octanes, give no doubly charged ions. This introduces striking individual differences among isomers that are not found in the lighter hydrocarbons. Individual differences, depending on structure, are the rule in singly charged ion spectra.

\section{Conclusion}

The study of doubly charged ion spectra is of interest in that it gives additional evidence as to the ionization process. Doubly charged ion peaks are, however, not very useful in the practical application of most spectra to chemical analysis, although it is essential to know the complete spectrum of every pure compound analysed. In the case of propadiene and propyne there is a striking difference in the doubly charged ions that affords the best basis for distinguishing the compounds. In nearly every other case the peaks are too small to be used in analysis.

\section{References}

[1] Catalog of mass spectral data, American Petroleum Institute, Project 44, National Bureau of Standards.

[2] J. A. Hipple, Jr., Phys. Rev. 53, 530 (1938).

[3] P. Kusch, A. Hustrulid, and John T. Tate, Phys. Rev. 52, 843 (1937).

[4] A. Hustrulid, P. Kusch, and John T. Tate, Phys. Rev. 54, 1037 (1938).

[5] American Petroleum Institute catalog of mass spectral data, description sheet 1 .

[6] J. Delfosse and Walker Bleakney, Phys. Rev. 56, 256 (1939).

[7] American Petroleum Institute catalog of mass spectral data, tables 57, 58, and 59 (Atlantic Refining Co., Philadelphia, Pa.)

[8] D. P. Stevenson and J. A. Hipple, J. Am. Chem. Soc. 64, 1588 (1942).

Washington, October 29, 1948 